\title{
Engajamento informacional nas redes sociais: como calcular?
}

\section{Informational engagement in online social networks: how to calculate?}

\author{
Ilaydiany Oliveira da Silva ${ }^{1}$, Fabio Castro Gouveia ${ }^{2}$ \\ ${ }^{1}$ Universidade Federal de Goiás (UFG), Goiânia, GO, Brasil. ORCID: https://orcid.org/0000-0002-3171-7878 \\ 2 Fundação Oswaldo Cruz (FiuCruz), Rio de Janeiro, RJ, Brasil. ORCID: https://orcid.org/0000-0002-0082-2392
}

Autor para correspondência/Mail to: Ilaydiany Oliveira da Silva, ilaydiany18@hotmail.com

Recebido/Submitted: 18 de setembro de 2020; Aceito/Approved: 21 de dezembro de 2020

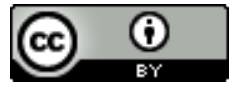

Copyright (c) 2021 Silva \& Gouveia. Todo o conteúdo da Revista (incluindo-se instruções, política editorial e modelos) está sob uma licença Creative Commons Atribuição 4.0 Internacional. Ao serem publicados por esta Revista, os artigos são de livre uso em ambientes educacionais, de pesquisa e não comerciais, com atribuição de autoria obrigatória. Mais informações em http://revistas.ufpr.br/atoz/about/submissions\#copyrightNotice.

\begin{abstract}
Resumo
Introdução: este estudo ressalta a inserção das redes sociais on-line no atual cenário informacional da sociedade, dando ênfase à importância da análise do engajamento informacional dos indivíduos nessas mídias sociais como forma de compreender o comportamento informacional da população mediante o acesso e uso das informações disponibilizadas por estes meios comunicacionais. Método: apresenta a proposta de fórmulas para o cálculo do engajamento informacional nas redes sociais on-line por meio do ajuste na quantificação das ferramentas de interação existentes nas mídias sociais como curtir, compartilhar e comentar. Assim, as fórmulas sugeridas atribuem pesos baseados na amostra em análise e que representam o nível de engajamento relativo de uma publicação nas redes sociais on-line. Resultados: comprova que as fórmulas apresentadas são passíveis de aplicação nas redes sociais on-line Facebook, Instagram, YouTube e Twitter por apresentar uma dinâmica de pesos para cada tipo de interação, refletindo assim o engajamento relativo realizado pelas pessoas em uma publicação para cada contexto analisado. Conclusão: contribui com o desenvolvimento de pesquisas voltadas para a análise de redes sociais on-line e possibilita uma melhor compreensão acerca dos fatores que envolvem o comportamento informacional do indivíduo mediante o uso das mídias sociais.
\end{abstract}

Palavras-chave: Engajamento informacional; Redes sociais; Comportamento informacional.

\begin{abstract}
Introduction: this study juts the insertion of online social networks in the current informational scenario of society, emphasizing the importance of analyzing the informational engagement of individuals in these social media as a way of understanding the informational behavior of the population through the access and use of the information made available by these communicational means. Method: it presents the proposed formulas for calculating informational engagement in online social networks by adjusting the quantification of existing interaction tools on social media such as like, share and comment. Thus, the suggested formulas assign weights based on the sample under analysis and which represent the level of relative engagement of a publication on online social networks. Results: it proves that the formulas presented are amenable to the application on the online social networks Facebook, Instagram, YouTube and Twitter for presenting a dynamic of weights for each type of interaction, thus reflecting the relative engagement made by people in a publication for each context analyzed. Conclusion: it contributes to the development of research aimed at analyzing online social networks and enables a better understanding of the factors that involve the individual's informational behavior through the use of social media.
\end{abstract}

Keywords: Informational engagement; Social networks; Informational Behavior.

\section{INTRODUÇÃO}

Os avanços tecnológicos têm acelerado de forma exponencial a construção de meios e suportes para produção, compartilhamento e acesso à informação. Dentre esses meios, destacam-se as redes sociais on-line, as quais são de fácil acesso a parte significativa da população, de simples manuseio e alcance geográfico das informações disponibilizadas.

As redes sociais on-line são conceituadas como "o ambiente digital organizado por meio de uma interface virtual própria (desenho/mapa de um conceito) que se organiza agregando perfis humanos que possuam afinidades, pensamentos e maneiras de expressão semelhantes e interesse sobre um tema comum" (Zenha, 2014, p. 10).

É possível perceber que em razão dessas redes sociais a sociedade tem mudado, cada vez mais, seu comportamento informacional no que concerne à busca, ao uso e à disseminação das informações. Essa mudança comportamental é reflexo da construção de um corpo social digitalmente mais participativo, característica que integra a web $2.0^{1}$ e permite a livre criação e disponibilização de materiais informacionais nessas estruturas interativas.

Essa mudança comportamental é confirmada ao se analisar os dados do Relatório Digital (2019) do site We Are Social $^{2}$, que apresenta uma lista com as 20 redes sociais on-line mais utilizadas no mundo, com destaque apenas para as cinco primeiras, que são o Facebook com 2.271 bilhões de usuários, YouTube (1,900 milhões), WhatsApp (1,500 milhões), Facebook Messenger (1,300 milhões) e o WBXIN/WeChat (1,083 milhões). No Brasil, o ranking

\footnotetext{
${ }^{1}$ Termo alcunhado por DiNucci (1999) para designar a web que permite interação social.

${ }^{2}$ https://wearesocial.com/
} 
das 10 redes sociais on-line é estabelecido por, respectivamente: YouTube, Facebook, WhatsApp, Instagram, Facebook Menssenger, Twitter, LinkedIn, Pinterest, Skype e Snapchat (Digital in 2019, 2019)³.

O quantitativo de redes sociais on-line espalhadas pela sociedade é enorme. Todas elas apresentam formas de interações entre os indivíduos. Em vista disso, define-se como tema deste estudo a padronização nos cálculos de engajamento informacional das redes sociais on-line. Desse modo, objetiva-se propor uma fórmula padronizada capaz de mensurar o engajamento informacional nas redes sociais on-line como forma de estabelecer um padrão metodológico, visando aprimorar as pesquisas desenvolvidas na área e contribuir com a qualidade das investigações que estão sendo desenvolvidas.

A escolha pela temática e seu aprofundamento se justifica por se buscar aferir, por meio da aplicação de métricas informacionais da área de Ciência da Informação, como a webmetria e Altmetria, as características e fatores suscetíveis a pesquisas direcionadas a determinados interesses, como estratégias de marketing e fonte de dados em métodos digitais, para estudos científicos de informações produzidas pela sociedade.

A definição de uma fórmula padrão para calcular o engajamento informacional contribuirá com as pesquisas científicas que buscam demonstrar e avaliar o comportamento informacional da sociedade frente às comunicações publicadas nas redes aludidas. Além disso, poupar o tempo dos pesquisadores que buscam uma metodologia para suas análises de engajamento informacional e dar maior credibilidade às pesquisas científicas brasileiras e internacionais que estão atuando com a temática em questão, das quais cita-se R. B. Silva (2013), I. C. O. Silva (2018), Alves, Trindade, de Souza, Mauro, e Baader (2018), Seco, dos Santos, e Bartalo (2016) e Presser e de Menezes (2016).

Isto posto, visando delimitar melhor as discussões, apresentar-se-á, a seguir, breve contexto teórico-conceitual acerca das redes sociais on-line e do engajamento informacional como forma de embasar a compreensão do assunto estudado.

\section{REDES SOCIAIS ON-LINE E ENGAJAMENTO INFORMACIONAL}

Para iniciar as discussões sobre redes sociais on-line e engajamento informacional torna-se oportuno compreender as relações terminológicas existentes nesse cenário, tais como redes sociais, mídias sociais e redes sociais on-line. Apesar dessas terminologias serem utilizadas como sinônimos pela sociedade, elas apresentam diferentes conceitos e finalidades.

A princípio, parte-se do conceito de Redes sociais, que "são elos existentes entre indivíduos que compartilham de um mesmo interesse e através de suas ligações em comum permitem que informações relevantes sejam repassadas entre os indivíduos daquela determinada rede" (I. O. Silva, 2016, p. 116).

Já as Mídias sociais podem ser compreendidas como um conglomerado de seis categorias de comunicação, das quais circundam os fóruns e quadros de mensagens, sites de crítica e opinião, marcadores sociais, compartilhamento de mídia, blogs e micro blogs e redes sociais (Sterne, 2011). São instrumentos para a criação de redes sociais entre indivíduos, por meio da formação de vínculos pautados por interesses comuns. Assim, as redes formadas on-line também são chamadas de redes sociais digitais (Souza \& Cardoso, 2011).

No tocante às redes sociais on-line ou redes sociais digitais, estas são conceituadas por Marteleto (2010) como o símbolo da construção das relações humanas na web e se configuram por meio de aplicativos e sites que permitem relações por meio de interações virtuais. Essas interações são relacionadas com as características que cada rede social on-line apresenta - formas de construção e disponibilização de conteúdo, recebimento e envio mensagens, visualizações, materiais a serem postados, público a ser atingido, dentre outros.

Para R. C. Recuero (2009), uma das características das atuais redes sociais on-line é a sua capacidade de difundir informações através das conexões existentes entre os atores da rede, ou seja, entre pessoas que integram o mesmo interesse informacional. Por intermédio de análises quali/quantitativas, os pesquisadores utilizam essas fontes com vistas a compreender o comportamento informacional de indivíduos ou mesmo contas institucionais, visto que "as redes sociais na Internet alteram de forma expressiva o fluxo de informações na sociedade" (R. C. Recuero, 2009, p. 25).

Cada rede social on-line oferece um conjunto de affordances - no português, recursos ou propriedades - que os meios disponibilizam para o usuário interagir de formas diversas. À vista disso, essas propriedades precisam ser consideradas dentro do contexto em que as ações dos usuários foram efetuadas e com peso relativo ao seu nível de interação. "uma rede social on-line não se forma pela simples conexão de terminais. Trata-se de um processo emergente que mantém sua existência através de interações entre os envolvidos" (Primo, 2007, p. 5).

Essas affordances direcionam o processo de interação e geração de conteúdos da rede social, espaço onde convencionalmente os usuários começam a seguir páginas de seu interesse social para se informar acerca de assuntos e interagir com outros utilizadores sobre o que é publicado nessas faces por meio de ferramentas de

\footnotetext{
${ }^{3}$ Documento não datado e não paginado
} 
interação, como as curtidas (likes) - que no Facebook se apresentam desde 2016 como um conjunto de opções (Like, Love, Haha, Wow, Sad e Angry, com adições eventuais temporárias como Thankful e mais recentemente Care) conhecidas também como reações ou reactions - comentários (comments) e compartilhamentos (shares), a essa tríade, damos o nome de 3C's.

As análises não se resumem apenas nessas três ferramentas, dado que todos os comportamentos dos usuários dentro de uma rede social on-line são passíveis de avaliação. De acordo com a DirectLabs (2009), dentre as métricas informacionais capazes de serem aplicadas nessas redes citam-se: visibilidade, influência, participação e engajamento.

A visibilidade diz respeito ao número de visitas e o tempo que o usuário permanece em cada página. A Influência mensura quantos outros ambientes estão referenciando a iniciativa ou publicação do utilizador, por meio da análise de links, menções ou marcações do perfil on-line. Quanto à participação, mede o quanto os usuários estão interagindo em determinada rede ou gerando conteúdo através de comentários, fotos publicadas, vídeos postados e outras maneiras de geração de conteúdo. E o engajamento se refere o quanto o público está interagindo com a marca e seus conteúdos (Macedo, 2014).

Dentre os quatro indicadores métricos mencionados, evidencia-se neste estudo o engajamento informacional, termo oriundo do ato de interagir, compartilhar informações entre usuários conectados à rede (Terra \& Carvalho, 2014) e que é composto pelo somatório da tríade dos 3C's.

A discussão parte do entendimento de que a análise da métrica de engajamento informacional não deve ser realizada pelo somatório das affordances, porque a simples soma não é capaz de representar de maneira equilibrada o engajamento em sua especificidade. Conforme R. Recuero (2014), as ferramentas "curtir", "compartilhar" e "comentar" são a base da conversação em rede que podem provocar implicações nas apropriações de capital social. A autora ressalta que o botão "curtir" é utilizado na comunicação sem precisar elaborar uma resposta. Há, então, uma interação mínima, pois o ator não necessariamente precisa ler tudo o que foi dito. Já o ícone "compartilhar" dá visibilidade para a publicação, de modo a ampliar o seu alcance na rede; lançar a divulgação de algo importante.

Por fim, R. Recuero (2014, p. 121) declara que:

O comentário, portanto, parece envolver um maior engajamento do ator com a conversação e um maior risco para a face, pois é uma participação mais visível. Isso porque aquilo que é dito pode ser facilmente descontextualizado quando migrar para outras redes através das ferramentas de compartilhamento, de curtida e mesmo de comentário.

Assim, diante da necessidade de um indicador de engajamento que leve em consideração as diferentes características que cada ferramenta das redes sociais on-line apresenta, este artigo foi desenvolvido com o intuito de discutir a funcionalidade de aplicação da fórmula de engajamento informacional nas redes sociais e sugerir uma fórmula padrão.

Desse modo, com o propósito de atingir esse objetivo, buscaram-se, por meio da literatura científica, estudos que sugerissem uma fórmula para definir o engajamento informacional nas redes sociais. Logo, identificou-se o estudo de Biancovilli, Picanço, e Jurberg (2017), que analisa o impacto das informações disponibilizadas no Facebook sobre câncer. As autoras concordam com a ideia apresentada por R. Recuero (2014) e definem a média ponderada de curtidas/reações ou reações, compartilhamentos e comentários a partir de uma fórmula com pesos definidos e fundamentados na relevância de cada ferramenta. Desse modo, definiram como indicador de engajamento o somatório dos 3C's, atribuindo um peso de 0,05 para curtidas; 0,2 para compartilhamentos; e 0,75 para comentários. O somatório dessas métricas definiria quais as publicações que proporcionam o maior engajamento na divulgação e impacto da informação sobre câncer.

No presente estudo, partiu-se da premissa de que o estabelecimento de pesos iria, a priori, ao encontro ao entendimento das diferentes formas e frequências em que ocorrem as apropriações das ferramentas de sites de mídias sociais. Assim, buscou-se estabelecer pesos para cada uma destas de forma não arbitrária, delimitando valores congruentes com a importância relativa de cada ferramenta e considerando-se a especificidade da amostra em análise, conforme descrito na seção a seguir.

\section{MÉTODO}

Com vistas a estabelecer uma fórmula aplicável às diferentes redes sociais on-line e ajustá-la às diferentes amostras, fez-se necessário, inicialmente, definir pesos para cada uma das ferramentas que compõem o conjunto em análise, para assim se determinar um valor de engajamento informacional em que se diferenciam as características de cada uma das affordances.

Para melhor definir a metodologia utilizou-se da tríade dos 3C's apresentada anteriormente. Nesse sentido, compreende-se que se faz necessário instituir o Engajamento Total da Amostra (ETA), que deve ser definido pela 
soma do total de cada item que compõe a tríade, ou seja, a soma das reações mais a soma dos compartilhamentos e a soma dos comentários constituem o ETA, conforme apresentado na fórmula abaixo:

$$
E T A=\sum \text { reações }+\sum \text { compartilhamentos }+\sum \text { comentários }
$$

Definido o ETA, partir-se-á para a definição dos pesos das 3C's. Percebe-se que o peso deve se ponderar a um terço da soma da ferramenta dividido pela ETA, conforme descrito na fórmula que segue.

$$
\begin{aligned}
\text { pesodasreações }(p r) & =\frac{1}{\sum \text { Reações } / E T A \times 3} \\
\text { pesodoscompartilhamentos }(\text { pcome }) & =\frac{1}{\sum \text { compartilhamentos } / E T A \times 3} \\
\text { pesodoscomentários }(\text { pcome }) & =\frac{1}{\sum \text { comentários } / E T A \times 3}
\end{aligned}
$$

Caso o somatório de uma das ferramentas seja igual a zero, considera-se o seu peso igual a zero. Após definir o peso de cada uma das ferramentas, encaminhar-se-á para a fórmula final do engajamento, que deve ser aplicada em cada uma das postagens e listados os valores das reações, compartilhamentos e comentários de determinada postagem. Logo após, cada uma deve ser multiplicada por seu respectivo peso, na qual a soma dessas três equações define o engajamento de cada postagem, conforme a Fórmula 5, apresentada abaixo.

$$
\text { Engajamento }=\text { reações } \times(\text { pr })+\text { compartilhamentos } \times(\text { pcomp })+\text { comentários } \times(\text { pcome })
$$

Assim, o engajamento informacional proposto perpassa por uma sequência de cinco fórmulas. Na Fórmula 1 são somadas todas as curtidas/reações, compartilhamentos e comentários de todas as postagens, em que obter-se-á o Engajamento Total da Amostra (ETA). Em seguida, são aplicadas as Fórmulas 2, 3 e 4, nas quais definir-se-ão os pesos para cada indicador, em que o peso se refere a um terço da soma da ferramenta dividido pela soma do engajamento. A partir dessa definição de pesos aplicar-se-á a fórmula 5 , em que cada ferramenta de cada postagem é multiplicada por seu respectivo peso, e a soma dessas três fórmulas finais definem o engajamento de cada postagem.

Sendo assim, os totais de comentários, curtidas/reações ou compartilhamentos representam um terço de cada indicador final gerado, valorizando as ações menos frequentes, porém que necessitam de maior índice na formação do indicador de engajamento informacional gerado.

Desse modo, tendo a relação alcançada pela aplicação das fórmulas é possível construir um ranking das postagens que causaram maior "impacto", baseado não somente em uma soma simples dos diferentes tipos de engajamento informacional, mas a partir de uma completude que leva em consideração algum tipo de peso para cada uma dessas ferramentas, fundamentada na frequência relativa destas na amostra em análise.

Em casos de redes sociais que não seja possível estabelecer a tríade, como é o caso do Instagram - que não permite a identificação e estratificação dos compartilhamentos, tendo acesso apenas aos dados referentes aos comentários e curtidas - sugere-se a aplicação das fórmulas com os dados das ferramentas existentes, conforme descrito nas fórmulas adaptadas abaixo: Em que:

$$
\text { EngajamentoTotaldaAmostra }=\sum \text { reações }+\sum \text { cometários }
$$

Assim:

$$
\begin{gathered}
\text { pesodasreações }(p r)=\frac{1}{\sum \text { Reações } / E T A \times 2} \\
\text { pesodoscomentários }(\text { pcome })=\frac{1}{\sum \text { comentários } / E T A \times 2}
\end{gathered}
$$

\section{Conclui-se que para cada postagem:}

Engajamento $=$ reações $\times(p r)+$ comentários $\times($ pcome $)$ 
A adaptação das fórmulas permite uma melhor adequação aos pesos das ferramentas existentes em cada rede social, sejam elas díade, tríade, tétrade, pentade, héxade ou demais, possibilitando uma melhor representação do engajamento informacional por meio do uso dos recursos utilizados.

Para a análise de aplicabilidade das fórmulas de engajamento propostas neste estudo, definiu-se coletar os dados relacionados às ferramentas compartilhar, comentar e curtir do perfil da "Web Conferência em Ciência da Informação e Biblioteconomia (WebConCIB)" na rede social Facebook ${ }^{4}$. Para tanto, coletaram-se os dados no período de 3 de abril a 15 de maio de 2020 e utilizou-se o software Facepager ${ }^{5}$, que é um OpenSource que busca e extrai postagens de redes sociais como Facebook, Twitter e YouTube com informações acerca das interações (curtidas, comentários e compartilhamentos) existentes em cada postagem. Os resultados da aplicação são apresentados na próxima seção.

\section{RESULTADOS}

Após coletar as 65 publicações referentes ao período apresentado anteriormente para o Facebook, identificou-se a quantidade de comentários, compartilhamentos e curtidas/reações das postagens. Os dados foram organizados, aplicados nas fórmulas e chegou-se aos seguintes valores:

$\sum$ reações $=735$

$\sum$ comentários $=49$

$\sum$ compartilhamentos $=517$

Fórmula $1 \mathrm{ETA}=735+49+517=>\mathrm{ETA}=1301$

Fórmulas 2, 3 e $4^{6}$ :

$$
\begin{gathered}
\text { pesodasreações }(\text { pr })=\frac{1}{\sum 735 / 1301 \times 3}=>(\text { pr })=0,59 \\
\text { pesodoscompartilhamentos }(\text { pcome })=\frac{1}{\sum 517 / 1301 \times 3}=>(\text { pcomp })=0,84 \\
\text { pesodoscomentários }(\text { pcome })=\frac{1}{\sum 49 / 1301 \times 3}=>(\text { pcomp })=8,85
\end{gathered}
$$

Aplicando a Fórmula 5 na primeira publicação de código PUB-FAC-29 da Tabela 1 verifica-se que

$$
\text { Engajamento }^{7}=43 \times 0,59+4 \times 0,84+10 \times 8,85=>\text { Engajamento }=117,23
$$

Na fórmula sugerida por Biancovilli et al. (2017), que é baseada no somatório das 3Cs, em que se atribui os pesos de 0,05 para curtidas/reações; 0,2 para compartilhamentos; e 0,75 para comentários, perceberam-se valores de engajamento relativamente baixos, com elevado número de engajamentos abaixo de 1 (um) nas publicações analisadas, conforme descrito na Tabela 1.

Com os dados apresentados aplicaram-se as duas metodologias discutidas no presente artigo: a primeira, sugerida pelos autores deste estudo (Engajamento com Pesos Calculados para Amostra); e a segunda proposta pelas autoras Biancovilli et al. (2017) (Engajamento com Pesos Pré-Definidos). Em seguida, estabeleceu-se um ranking com as 65 postagens, elencando as diferenças encontradas nos resultados, as quais foram identificadas por códigos estabelecidos pelos autores.

\footnotetext{
${ }^{4}$ https://www.facebook.com/webconcib

${ }^{5}$ https://github.com/strohne/Facepager

${ }^{6}$ Os valores dos pesos foram arredondados à segunda casa decimal para facilitar a visualização, mas nos cálculos utilizou-se o valor sem arredondamento

${ }^{7}$ Os valores de engajamento também foram arredondados à segunda casa decimal
} 


\begin{tabular}{|c|c|c|c|c|c|c|}
\hline Rank & Publicação & Comentários & Reações & Compartilhamentos & $\begin{array}{l}\text { Engajamento } \\
\text { PCA }\end{array}$ & $\begin{array}{c}\text { Engajamento } \\
\text { PPD }\end{array}$ \\
\hline 1 & PUB-FAC-29 & 10 & 43 & 4 & 117,23 & 10,45 \\
\hline 2 & PUB-FAC-41 & 6 & 28 & 52 & 113,24 & 16,30 \\
\hline 3 & PUB-FAC-54 & 7 & 21 & 10 & 82,73 & 8,30 \\
\hline 4 & PUB-FAC-14 & 4 & 19 & 29 & 70,94 & 9,75 \\
\hline 5 & PUB-FAC-02 & 3 & 18 & 37 & 68,21 & 10,55 \\
\hline 6 & PUB-FAC-03 & 1 & 13 & 49 & 57,62 & 11,20 \\
\hline 7 & PUB-FAC-28 & 0 & 36 & 43 & 57,31 & 10,40 \\
\hline 8 & PUB-FAC-47 & 2 & 18 & 28 & 51,81 & 8,00 \\
\hline 9 & PUB-FAC-08 & 3 & 7 & 9 & 38,23 & 4,40 \\
\hline 10 & PUB-FAC-37 & 0 & 19 & 30 & 36,37 & 6,95 \\
\hline 11 & PUB-FAC-05 & 1 & 9 & 17 & 28,42 & 4,60 \\
\hline 12 & PUB-FAC-01 & 0 & 18 & 21 & 28,24 & 5,10 \\
\hline 13 & PUB-FAC-58 & 0 & 13 & 23 & 26,96 & 5,25 \\
\hline 14 & PUB-FAC-36 & 2 & 8 & 3 & 24,94 & 2,50 \\
\hline 15 & PUB-FAC-12 & 0 & 18 & 16 & 24,04 & 4,10 \\
\hline 16 & PUB-FAC-22 & 0 & 18 & 14 & 22,36 & 3,70 \\
\hline 17 & PUB-FAC-59 & 0 & 12 & 18 & 22,18 & 4,20 \\
\hline 18 & PUB-FAC-18 & 1 & 14 & 6 & 22,14 & 2,65 \\
\hline 19 & PUB-FAC-24 & 1 & 15 & 4 & 21,06 & 2,30 \\
\hline 20 & PUB-FAC-52 & 2 & 4 & 1 & 20,90 & 1,90 \\
\hline 21 & PUB-FAC-60 & 0 & 4 & 18 & 17,46 & 3,80 \\
\hline 22 & PUB-FAC-38 & 1 & 11 & 2 & 17,02 & 1,70 \\
\hline 23 & PUB-FAC-39 & 0 & 15 & 7 & 14,72 & 2,15 \\
\hline 24 & PUB-FAC-06 & 1 & 8 & 1 & 14,41 & 1,35 \\
\hline 25 & PUB-FAC-57 & 1 & 8 & 1 & 14,41 & 1,35 \\
\hline 26 & PUB-FAC-34 & 1 & 5 & 3 & 14,32 & 1,60 \\
\hline 27 & PUB-FAC-65 & 0 & 17 & 4 & 13,39 & 1,65 \\
\hline 28 & PUB-FAC-04 & 1 & 7 & 0 & 12,98 & 1,10 \\
\hline 29 & PUB-FAC-26 & 0 & 12 & 7 & 12,95 & 2,00 \\
\hline 30 & PUB-FAC-51 & 0 & 14 & 5 & 12,45 & 1,70 \\
\hline 31 & PUB-FAC-19 & 1 & 6 & 0 & 12,39 & 1,05 \\
\hline 32 & PUB-FAC-56 & 0 & 15 & 4 & 12,21 & 1,55 \\
\hline 33 & PUB-FAC-42 & 0 & 14 & 4 & 11,62 & 1,50 \\
\hline 34 & PUB-FAC-49 & 0 & 15 & 3 & 11,37 & 1,35 \\
\hline 35 & PUB-FAC-62 & 0 & 13 & 4 & 11,03 & 1,45 \\
\hline 36 & PUB-FAC-33 & 0 & 12 & 4 & 10,44 & 1,40 \\
\hline 37 & PUB-FAC-20 & 0 & 13 & 3 & 10,19 & 1,25 \\
\hline 38 & PUB-FAC-64 & 0 & 15 & 1 & 9,69 & 0,95 \\
\hline 39 & PUB-FAC-44 & 0 & 13 & 2 & 9,35 & 1,05 \\
\hline 40 & PUB-FAC-16 & 0 & 12 & 2 & 8,76 & 1,00 \\
\hline 41 & PUB-FAC-61 & 0 & 13 & 1 & 8,51 & 0,85 \\
\hline 42 & PUB-FAC-63 & 0 & 10 & 3 & 8,42 & 1,10 \\
\hline 43 & PUB-FAC-07 & 0 & 4 & 7 & 8,23 & 1,60 \\
\hline 44 & PUB-FAC-35 & 0 & 8 & 3 & 7,24 & 1,00 \\
\hline 45 & PUB-FAC-10 & 0 & 9 & 2 & 6,99 & 0,85 \\
\hline 46 & PUB-FAC-31 & 0 & 9 & 2 & 6,99 & 0,85 \\
\hline 47 & PUB-FAC-30 & 0 & 10 & 1 & 6,74 & 0,70 \\
\hline 48 & PUB-FAC-45 & 0 & 10 & 1 & 6,74 & 0,70 \\
\hline 49 & PUB-FAC-13 & 0 & 11 & 0 & 6,49 & 0,55 \\
\hline 50 & PUB-FAC-43 & 0 & 6 & 3 & 6,06 & 0,90 \\
\hline 51 & PUB-FAC-55 & 0 & 10 & 0 & 5,90 & 0,50 \\
\hline 52 & PUB-FAC-21 & 0 & 7 & 0 & 4,13 & 0,35 \\
\hline 53 & PUB-FAC-09 & 0 & 5 & 1 & 3,79 & 0,45 \\
\hline 54 & PUB-FAC-15 & 0 & 5 & 1 & 3,79 & 0,45 \\
\hline
\end{tabular}




\begin{tabular}{|c|c|c|c|c|c|c|}
\hline Rank & Publicação & Comentários & Reações & Compartilhamentos & $\begin{array}{c}\text { Engajamento } \\
\text { PCA }\end{array}$ & $\begin{array}{c}\text { Engajamento } \\
\text { PPD }\end{array}$ \\
\hline 55 & PUB-FAC-27 & 0 & 5 & 1 & 3,79 & 0,45 \\
\hline 56 & PUB-FAC-23 & 0 & 6 & 0 & 3,54 & 0,30 \\
\hline 57 & PUB-FAC-32 & 0 & 6 & 0 & 3,54 & 0,30 \\
\hline 58 & PUB-FAC-40 & 0 & 5 & 0 & 2,95 & 0,25 \\
\hline 59 & PUB-FAC-17 & 0 & 3 & 1 & 2,61 & 0,35 \\
\hline 60 & PUB-FAC-46 & 0 & 3 & 1 & 2,61 & 0,35 \\
\hline 61 & PUB-FAC-25 & 0 & 3 & 0 & 1,77 & 0,15 \\
\hline 62 & PUB-FAC-11 & 0 & 2 & 0 & 1,18 & 0,10 \\
\hline 63 & PUB-FAC-50 & 0 & 2 & 0 & 1,18 & 0,10 \\
\hline 64 & PUB-FAC-53 & 0 & 2 & 0 & 1,18 & 0,10 \\
\hline 65 & PUB-FAC-48 & 0 & 1 & 0 & 0,59 & 0,05 \\
\hline
\end{tabular}

Tabela 1. Ranking de engajamento informacional das publicações no Facebook da WebConCIB por meio da fórmula com pesos calculados para amostra (PCA) comparado ao com pesos pré-definidos (PPD) de Biancovilli et al. (2017).

Fonte: dados da pesquisa (2020).

Ao analisar os resultados apresentados, é possível depreender que as notícias, apesar de se apresentarem em posições próximas, não comporiam o ranking na mesma ordem. Na fórmula apresentada neste estudo, os compartilhamentos são relativamente menos favorecidos e os comentários mais favorecidos em relação ao que estabelece os pesos atribuídos por Biancovilli et al. (2017). Em razão do cálculo equilibrar a importância dada a cada affordance, favorecendo as que tiveram menor frequência na amostra, caso fosse colocar no mesmo formato de peso atribuído pelas autoras ter-se-ia 0,86 para os comentários (contra 0,75); 0,08 para os compartilhamentos (contra 0,20); e 0,06 para as curtidas/reações (contra 0,05).

É importante ressaltar que a fórmula proposta gera pesos que são dependentes do que for encontrado na amostra em estudo, equilibrando, assim, a característica de apropriação das affordances ocorrida para aquele conjunto de postagens. Neste sentido, a importância atribuída às formas de interação (curtidas/reações, compartilhamentos ou comentários) dar-se-á de acordo com a frequência existente na amostra temática, o que equilibrará os índices de interação dos indivíduos ao utilizarem cada uma dessas ferramentas.

Isto posto, considera-se que o cálculo do engajamento informacional permite solucionar o problema identificado quanto a correta quantificação do engajamento informacional, permitindo uma padronização da metodologia a ser aplicada nas pesquisas em redes sociais on-line.

\section{CONSIDERAÇÕES FINAIS}

A proposta dessa forma de cálculo se difere da apresentada por Biancovilli et al. (2017), por se tratar de uma definição de valores para ajustes não arbitrários e coerentes com o valor do engajamento das ferramentas de interação com as postagens no Facebook ou de outros sites de mídias sociais relativos ao conjunto de dados em análise. No caso dos comentários que representam um maior valor informacional quanto à exposição de ideias e troca de conhecimento, por exemplo, estes geralmente apresentam quantitativos mais baixos quando comparados às formas mais simples de reagir a uma publicação ou clicar para compartilhar dada notícia. Sendo assim, o cálculo aqui proposto visa enfatizar o valor dos eventos menos frequentes nas amostras, equilibrando, com isso, a pontuação de engajamento realizado nas postagens quanto à sua forma.

Ao atribuir pesos a cada um dos componentes do engajamento na mídia social, relativizando sua importância baseado na frequência em que os mesmos ocorrem em dada amostra, tem-se uma correção do engajamento originalmente proposto pelos sites de mídias sociais onde se efetua uma mera soma dos componentes sem qualificar seus diferentes níveis de envolvimento, cujo resultado é dependente das características do conjunto de postagens em estudo. Ainda, por se tratar de um cálculo definido e que não privilegia, a priori, nenhuma categoria de engajamento, mantém-se a neutralidade na atribuição dos pesos que cada um desses elementos deve ter. As fórmulas também são adaptáveis aos diferentes sites de redes sociais, conforme exemplificado para duas delas.

A relevância científica deste estudo situou-se no aprimoramento das pesquisas desenvolvidas na área por meio da contribuição de métodos e procedimentos adequados à elaboração de estudos voltados para a quantificação de informações nas redes sociais e o engajamento da sociedade mediante novas formas de comunicação que estão ocorrendo no meio virtual. Assim, a possibilidade de se analisar adequadamente o engajamento informacional existente nas redes sociais favorece o entendimento do comportamento informacional que sucede na esfera virtual, proporcionando investigações mais detalhadas sobre a produção, acesso e uso das informações.

Por conseguinte, a aplicação da fórmula em estudos posteriores será de importância para pesquisas realizadas na área, de modo a contribuir com o melhor entendimento da dimensão dos diferentes tipos de interações 
presentes em sites de redes sociais e favorecer o desenvolvimento de novas análises voltadas para o engajamento informacional da sociedade por meio da aplicação aqui apresentada.

\section{AGRADECIMENTOS}

Este trabalho foi realizado com apoio da Coordenação de Aperfeiçoamento de Pessoal de Nível Superior - Brasil (CAPES) - Código de Financiamento 001 e Conselho Nacional de Desenvolvimento Científico e Tecnológico, Processo 430982/2018-6, chamada universal MCTIC/CNPQ n²8/2018 e as instituições UFG, UFRN e FIOCRUZ pela parceria de seus pesquisadores. 


\section{REFERÊNCIAS}

Alves, M. C. D., Trindade, E., de Souza, L. S., Mauro, R., \& Baader, C. (2018). Isso foi compartilhado com sucesso: questões de consumo midiático dos jovens paulistanos no facebook. Comunicação \& Informação, 21(1), 109-125. doi: 10.5216/ci.v21i1.49914

Biancovilli, P., Picanço, L., \& Jurberg, C. (2017). To read or not to read? identifying communication patterns in three cancer-related facebook pages. Cogent Social Sciences, 3(1), 1331816. Recuperado de https://www.cogentoa.com/article/ 10.1080/23311886.2017.1331816.pdf

Digital in 2019. (2019). Recuperado de https://wearesocial .com/global-digital-report-2019

DiNucci, D. (1999). Fragmented future. Print, 53(4), 32-33. Recuperado de http://darcyd.com/fragmented_future.pdf

DirectLabs. (2009). Faq sobre mídias sociais. Recuperado de http://www.directlabs.com.br

Macedo, T. (2014). Métricas de marketing digital sua aplicação nas aãões de marketing das organizaa̧ões: estudo de caso múltiplos. 2014 (Dissertação de mestrado). Universidade federal do Rio Grande do Sul, Porto Alegre, Brasil.

Marteleto, R. M. (2010). Redes sociais, mediação e e apropriação de informaąões: situando campos, objetos e conceitos na pesquisa em ciência da informação. Pesquisa Brasileira em Ciência da Informação, 3(1), 27-46.

Presser, N. H., \& de Menezes, P. R. A. (2016). Conteúdo informacional gerado nas redes sociais: o universo dos parques de diversão. InCID: Revista de Ciência da Informação e Documentação, 7(2), 67-92. doi: 10.11606/issn.21782075.v7i2p67-92

Primo, A. (2007). O aspecto relacional das interações na web 2.0. In E-compós (v. 9, p. 1-21).

Recuero, R. (2014). Curtir, compartilhar, comentar: trabalho de face, conversação e redes sociais no facebook. Verso e reverso, 28 (68), 117-127. doi: 10.4013/ver.2014.28.68.06

Recuero, R. C. (2009). Redes sociais na internet. Porto Alegre: Sulina.

Seco, L. F. C., dos Santos, Z. P., \& Bartalo, L. (2016). Comportamento informacional e compartilhamento da informação no instagram information behavior end information share of instagram. Revista ACB: Biblioteconomia em Santa Catarina, 21(1), 46-60. Recuperado de http://hdl.handle.net/20.500.11959/brapci/76475

Silva, I. C. O. (2018). Rastreando epidemias na web: uma análise do comportamento informacional da sociedade brasileira mediante das epidemias de dengue, chikungunya e zika a partir do método webmétrico (Tese de doutorado, Universidade Federal do Rio de Janeiro, Rio de Janeiro, Brasil). Recuperado de https://ridi.ibict.br/bitstream/ 123456789/997/1/Ilaydiany\%20Cristina\%20Oliveira\% 20da\%20Silva_Doutorado_2018.pdf

Silva, I. O. (2016). A memória social registrada no facebook. Revista Conhecimento em Ação. Rio de Janeiro, 1(1). Recuperado de http://hdl.handle.net/20.500.11959/brapci/ 71079

Silva, R. B. (2013). Mídias sociais e política: as jornadas de junho no facebook do pt. Comunicação $\mathscr{G}$ Informação, 16(2), 53-71. doi: 10.5216/ci.v16i2.27465

Souza, C. H. M., \& Cardoso, C. (2011). As redes sociais digitais: um mundo em transformação. Agenda Social, 5, 6578. Recuperado de http://www.uenf.br/Uenf/Downloads/
Agenda_Social_8427_1312371250.pdf

Sterne, J. (2011). Métricas em mídias sociais. São Paulo: Nobel.

Terra, C. F., \& Carvalho, E. (2014). Street art: das margens da sociedade para a comunicação organizacional: o grafite no ambiente digital como técnica de branding para relacionamento com o público consumidor metropolitano. In Anais do $14^{\circ}$ congresso internacional de relaãóes públicas e comunicação. Salvador: ALARP.

Zenha, L. (2014). Redes sociais online: o que são as redes sociais e como se organizam? Caderno de Educação, 19(48), 09-23. Recuperado de https://www.researchgate.net/ publication/323642463_12_-_Redes_sociais_online_o _que_sao_as_redes_sociais_e_como_se_organizam _revisado_com_palavras_chave/references
Como citar este artigo (APA):

Silva, I. O. \& Gouveia, F. C. (2021). Engajamento informacional nas redes sociais: como calcular?. AtoZ: novas práticas em informação e conhecimento, 10(1), 94 - 102. Recuperado de: http://dx.doi.org/10.5380/ atoz.v10i1.76633 